\title{
xCT expression modulates cisplatin resistance in Tca8113 tongue carcinoma cells
}

\author{
PENG ZHANG, WEI WANG, ZHENHUI WEI, LI XU, XUANNING YANG and YUANHONG DU \\ Department of Stomatology, No. 463 Hospital of Chinese PLA, Shenyang, Liaoning 110042, P.R. China
}

Received March 26, 2015; Accepted April 19, 2016

DOI: $10.3892 / 01.2016 .4571$

\begin{abstract}
Tongue squamous cell carcinoma (TSCC), which is a subtype of head and neck cancer, is the most common type of oral cancer. Due to its high recurrence rate and chemoresistance, the average survival rate for patients with TSCC remains unsatisfactory. At present, cisplatin (CDDP) is utilized as the first-line treatment for numerous solid neoplasms, including TSCC. CDDP resistance develops in the majority of patients; however, the mechanism of such resistance remains unknown. Therefore, the present study aimed to clarify the mechanism of CDDP resistance and attempted to reduce chemoresistance. The results indicated that CDDP significantly increased expression of $\mathrm{xCT}$, which is the light chain and functional subunit of the glutamate/cysteine transporter system $\mathrm{x}_{\mathrm{c}}{ }^{-}$, and a subsequent increase in glutathione (GSH) levels was observed. The present study demonstrated that the upregulation of $\mathrm{xCT}$ expression and intercellular GSH levels contributed to CDDP resistance in TSCC cells. Furthermore, xCT suppression, induced by small interfering RNA or pharmacological inhibitors, sensitized TSCC cells to CDDP treatment. In conclusion, the present study revealed that CDDP-induced $\mathrm{xCT}$ expression promotes CDDP chemoresistance, and $\mathrm{xCT}$ inhibition sensitizes TSCC cells to CDDP treatment. These results provide a novel insight into the molecular mechanisms involved in TSCC cell chemoresistance.
\end{abstract}

Correspondence to: Dr Yuanhong Du, Department of Stomatology, No. 463 Hospital of Chinese PLA, 46 Xiaoheyan Road, Dadong, Shenyang, Liaoning 110042, P.R. China

E-mail: yuanhongdu0326@163.com

Abbreviations: TSCC, tongue squamous cell carcinoma; CDDP, cisplatin; Nrf2, nuclear factor erythroid 2-related factor 2; ATF4, activating transcription factor 4; SASP, sulfasalazine; GSH, glutathione; ER, endoplasmic reticulum; ARE, antioxidant response element; AARE, amino acid response element

Key words: tongue carcinoma cells, cisplatin, $\mathrm{xCT}$, sulfasalazine, Nrf2, ATF4

\section{Introduction}

Tongue carcinoma, which is a subtype of head and neck cancer, is the most frequently occurring oral cancer, of which , 90\% is tongue squamous cell carcinoma (TSCC) (1). Currently, TSCC is the tenth most common solid neoplasm worldwide, with a comparatively low median survival rate (5-year survival rate, $\sim 50 \%$ ) (2). Recent studies have reported that chemotherapy decreases tumor size and reduces distant metastasis in TSCC patients $(3,4)$. Cisplatin (CDDP) is an efficacious antineoplastic compound, which is used for the treatment of a wide range of solid neoplasms, including non-small cell lung, ovarian, bladder, and head and neck carcinoma (5-8). However, the cytotoxicity caused by CDDP in TSCC chemotherapy remains unsatisfactory (9), and the exact mechanisms of CDDP-induced cytotoxicity have not been identified to date. CDDP resistance is the major obstacle that prevents successful treatment of TSCC (10). Recently, it has been reported that not only DNA-damaging stress but also endoplasmic reticulum (ER) and oxidative stress are involved in the therapeutic effect of CDDP $(11,12)$. In particular, TSCC cells with high glutathione (GSH) levels exhibit marked resistance to CDDP treatment (13). Thus, the association between CDDP resistance and GSH-associated pathways requires investigation.

GSH is a triple peptide molecule consisting of cysteine, glutamic acid and glycine residues that is critical for the maintenance of the intracellular redox balance and detoxification (14). As a result, GSH levels exhibit a positive correlation with chemotherapy resistance, including resistance to CDDP $(14,15)$. Numerous studies have revealed that CDDP resistance is associated with increased GSH levels and decreased reactive oxygen species (ROS) levels (15-17). However, the association between increased GSH levels and CDDP treatment, as well as CDDP-induced cytotoxicity in tongue carcinoma cells, remains unclear.

Cysteine is predominantly captured from the extracellular environment by system $\mathrm{x}_{\mathrm{c}}{ }_{\mathrm{c}}$ and it functions as the essential raw material for intercellular GSH synthesis (15). System $\mathrm{x}_{\mathrm{c}}{ }^{-}$consists of a light chain, $\mathrm{xCT} /$ solute carrier family $7 \mathrm{~A} 11$, and its cell surface subunit $4 \mathrm{~F} 2 \mathrm{hc} /$ cluster of differentiation (CD)98 (18). The specific function of system $\mathrm{x}_{\mathrm{c}}{ }^{-}$is determined by $\mathrm{xCT}(18)$ and thus, intracellular GSH levels are closely associated with the expression and function of xCT (19). Increasing evidence has revealed that $\mathrm{xCT}$ is expressed in various malignant tumors, and its expression is associated 
with the development of preneoplastic lesions and cancer, poor prognosis and drug resistance (20-23). Notably, xCT is important in maintaining high levels of GSH and contributes to CDDP resistance of ovarian cancer cell lines (15). In addition, $\mathrm{xCT}$ is markedly upregulated in resected tongue carcinoma specimens (24). Ye et al (19) demonstrated that proteasome inhibitor-induced $\mathrm{xCT}$ expression is positively modulated by the activation of nuclear factor erythroid 2-related factor 2 (Nrf2) and activating transcription factor 4 (ATF4) via the antioxidant response element (ARE) and amino acid response element (AARE) on the promoter human $x C T$ gene (19). In addition, Nrf2 and ATF4 are also induced by $\operatorname{CDDP}(25,26)$, while the mechanism of CDDP-inducible $\mathrm{xCT}$ expression and the association between that and CDDP resistance of TSCC cells remains unclear.

A series of compounds exhibit $\mathrm{xCT}$ inhibition, including the substrate inhibitor glutamine acid and the non-substrate inhibitor sulfasalazine (SASP) (27). SASP, a sulfa immunosuppressant that is widely used in the treatment of rheumatoid arthritis and inflammatory bowel diseases, is also an effective pharmacological inhibitor of $\mathrm{xCT}$ (28). Combined treatment with SASP increases the efficacy of several chemotherapeutic drugs, including gemcitabine, 5-fluouracil, bortezomib and doxorubicin, to lung adenocarcinoma cells $(19,29,30)$. SASP has also been reported to decrease CDDP resistance in small cell lung cancer cell lines (31). However, further study regarding the combined effect of SASP and CCDP on tongue carcinoma cell lines is required.

In the present study, the mechanism of CDDP-inducible $\mathrm{xCT}$ expression and the function of $\mathrm{xCT}$ upregulation in CDDP resistance in the Tca8113 tongue carcinoma cell line were investigated. $\mathrm{xCT}$ expression was robustly induced by CDDP in an Nrf2 and ATF4 activation-dependent manner. CDDP-induced cells death was increased when $\mathrm{xCT}$ was suppressed by small interfering RNA (siRNA) or its pharmacological inhibitor. These results indicate that CDDP-prompted $\mathrm{xCT}$ activation increases the resistance of tongue cancer cells to CDDP treatment, and the combination of CDDP and $\mathrm{xCT}$ inhibitor could benefit tongue cancer chemotherapy.

\section{Materials and methods}

Cell line. The human TSCC cell line Tca8113 was obtained from the China Center for Type Culture Collection (Wuhan, China). Tca8113 cells were cultured in RPMI-1640 medium (Sigma-Aldrich, St. Louis, MO, USA) containing 10\% fetal calf serum (Gibco; Thermo Fisher Scientific, Inc., Waltham, MA, USA) supplemented with $100 \mathrm{U} / \mathrm{ml}$ penicillin and $100 \mu \mathrm{g} /$ $\mathrm{ml}$ streptomycin (Life Technologies; Thermo Fisher Scientific, Inc.) at $37^{\circ} \mathrm{C}$ with in an atmosphere of $5 \% \mathrm{CO}_{2}$.

Reagents. CDDP and SASP (Sigma-Aldrich) were dissolved in 100\% dimethyl sulfoxide (DMSO; cat. no. 046-21981; Wako Pure Chemical Industries, Ltd., Osaka, Japan) and diluted to the appropriate concentrations (CDDP, $20 \mathrm{mg} / \mathrm{ml}$ and SASP, $300 \mathrm{mM}$ ) with culture medium prior to each experiment. The final DMSO concentration was $<0.1 \%$ for the cell experiments. Rabbit polyclonal anti-xCT antibody (cat. no. ab37185) was purchased from Abcam (Cambridge, MA, USA). Anti-4F2hc/CD98 (cat. no. sc-9160) and anti- $\beta$-actin antibodies (cat. no. sc-47778) were obtained from Santa Cruz Biotechnology, Inc. (Dallas, TX, USA). Rabbit polyclonal anti-CD44 variant (v) antibody (cat. no. 3578) was purchased from Cell Signaling Technology, Inc. (Danvers, MA, USA).

Reverse transcription-quantitative polymerase chain reaction $(R T-q P C R)$. Total RNA was isolated from Tca8113 cells using the Total RNA Purification kit (Norgen Biotek Corporation, Thorold, ON, Canada), according to the manufacturer's protocol. Complementary DNA was obtained from $1 \mu \mathrm{g}$ total RNA using iScript cDNA Synthesis kit (Bio-Rad Laboratories, Inc., Hercules, CA, USA). RT-qPCR analyses were performed using the iTaq Universal SYBR Green kit (Bio-Rad Laboratories, Inc.) and CFX Real-Time PCR Detection System (Bio-Rad Laboratories, Inc.), under the following cycling conditions: $98^{\circ} \mathrm{C}$ for $1 \mathrm{~min}$, followed by 45 cycles at $98^{\circ} \mathrm{C}$ for $5 \mathrm{sec}$ and $60^{\circ} \mathrm{C}$ for $30 \mathrm{sec}$. The designed RT-qPCR primers (Sigma-Aldrich) were as follows: Forward (F), 5'-GCTGTGATATCCCTGGCATT-3' and reverse (R), 5'-GGCGTCTTTAAAGTTCTGCG-3' for xCT; F, 5'-CCAGGTTCGGGACATAGAGA-3' and R, 5'-GAG CCTTGCCTGAGACAAAC-3' for 4F2hc/CD98; F, 5'-AGA AGGTGTGGGCAGAAGAA-3' and R, 5'-AAATGCACCATT TCCTGAGA-3' for CD44v; F, 5'-CGGTATGCAACAGGA CATTG-3' and R, 5'-ACTGGTTGGGGTCTTCTGTG-3' for Nrf2; F, 5'-CTTACGTTGCCATGATCCCT-3' and R, 5'-GAG AACACCTGGAGATGGGA-3' for ATF4; and F, 5'-TGAAGG TCGGAGTCAACGATTTGGT-3' and R, 5'-GAAGATGGT GATGGGATTTC-3' for glyceraldehyde-3-phosphate dehydrogenase (GAPDH). GAPDH was used as an internal control, and the expression of the target gene was normalized relative to the expression of GAPDH. Non-specific amplification and primer dimers were examined by dissociation curves at the end of the PCRs. Data was assessed according to the comparative $\mathrm{Cq}$ method $\left(2^{-\Delta \Delta \mathrm{Cq}}\right)(19)$.

Western blot analysis. Tca8113 cells were seeded at a density of $1.5 \times 10^{5}$ cells/well in 12 -well plates (cat. no. CLS3513; Sigma-Aldrich), and $24 \mathrm{~h}$ later, cells were lysed with CelLytic M Cell Lysis Reagent (Sigma-Aldrich) for whole-cell protein extraction. Genomic DNA was sonicated for $15 \mathrm{sec}$ with an output frequency of $20 \mathrm{kHz}$ and $50 \%$ amplitude, using a 150VT sonicator (Biologics, Inc, Cary, NC, USA). Protein concentration was measured using a Bicinchoninic Acid Protein Assay kit (Thermo Fisher Scientific, Inc.). Sample buffer (4X) and 1\% $\beta$-mercaptoethanol ( $\beta$-ME; Wako Pure Chemical Industries, Ltd.) were added to equivalent amounts of protein $(10 \mu \mathrm{g})$, and the samples were incubated at $95^{\circ} \mathrm{C}$ for $5 \mathrm{~min}$. The samples were next subjected to electrophoresis on an $8 \%(\mathrm{v} / \mathrm{v})$ sodium dodecyl sulfate-polyacrylamide gel (cat. no. WT0081BOX; Thermo Fisher Scientific, Inc.), and transferred to a polyvinylidene difluoride hybridization transfer membrane (EMD Millipore, Billerica, MA, USA). The membranes were blocked at room temperature with Blocker BLOTTO Blocking Buffer (Thermo Fisher Scientific, Inc.), washed 3 times with Tris-buffered saline containing $0.05 \%$ Tween (TBST; cat. no. 206-19131; Wako Pure Chemical Industries, Ltd.), and incubated with anti-xCT, anti-4F2hc, anti-CD44v and anti- $\beta$-actin antibodies overnight at $4{ }^{\circ} \mathrm{C}$. All the above primary antibodies were diluted in 1:1,000 with $1 \mathrm{X}$ TBST with $5 \%$ bovine serum albumin (cat. no. A9418; Sigma-Aldrich). Following 
3 washes with TBST, the membranes were incubated for $1 \mathrm{~h}$ at room temperature with goat anti-rabbit immunoglobulin (Ig)G-horseradish peroxidase-(HRP) (cat. no. sc-2054; Santa Cruz Biotechnology, Inc.) and goat anti-mouse IgG-HRP (cat. no. sc-2005; Santa Cruz Biotechnology, Inc.) secondary antibodies diluted 1:10,000 with 1X TBST, and visualized in a ChemiDoc MP system (Bio-Rad Laboratories, Inc.) using the ImmunoStar LD chemiluminescence system (Wako Pure Chemical Industries, Ltd.).

Plasmid construction and luciferase activity assay. Human $\mathrm{xCT}$ gene promoter-luciferase wild-type and mutant reporter plasmids were constructed as reported previously (19). For the luciferase activity assay, Tca8113 cells were seeded in 12 -well plates at a density of $0.5 \times 10^{5}$ cells/well for $24 \mathrm{~h}$ prior to transfection. The cells were co-transfected with $0.9 \mu \mathrm{g}$ luciferase reporter plasmids and $0.1 \mu \mathrm{g}$ pRL-TK (internal control; cat. no. E2241; Promega Corporation, Madison, WI, USA) using Lipofectamine LTX Reagent (Life Technologies; Thermo Fisher Scientific, Inc.). The luciferase activity assay was performed $24 \mathrm{~h}$ subsequent to transfection using the Dual-Luciferase ${ }^{\circledR}$ Reporter Assay System (Promega Corporation).

Intracellular GSH level determination. GSH was derivatized using monobromobimane (mBBr; Life Technologies; Thermo Fisher Scientific, Inc.) and separated by reverse-phase high-performance liquid chromatography as previously described (32). Briefly, Tca8113 cells were collected with phosphate-buffered saline (cat. no. 1662403; Bio-Rad Laboratories, Inc.) and lysed in 0.2 M 5-sulfosalicylic acid (cat. no. 197-04582; Wako Pure Chemical Industries, Ltd.) on ice for $10 \mathrm{~min}$. Samples were separated by centrifugation at $8,000 \mathrm{x} g$ for $5 \mathrm{~min}$. Next, $\mathrm{mBBr}$ was added to the supernatant fraction and allowed to react in the dark at room temperature for $30 \mathrm{~min}$, and then the absorbance at $490 \mathrm{~nm}$ was detected with an iMark ${ }^{\mathrm{TM}}$ Microplate Absorbance Reader (Bio-Rad Laboratories, Inc.). The precipitate was diluted in $0.1 \mathrm{~N} \mathrm{NaOH}$ (cat. no. 1310-73-2; Wako Pure Chemical Industries, Ltd.), and protein determination was performed using the Pierce Coomassie (Bradford) Protein Assay kit (Thermo Fisher Scientific, Inc.). Bovine gamma globulin (cat. no. 5000208; Bio-Rad Laboratories, Inc.) was used for the standard curve.

siRNA transfection of Tca8113 cells. Human Nrf2 siRNA\#1 (cat. no. 107966) and \#2 (cat. no. 115762); human ATF4 siRNA \#1 (cat. no. 122168) and \#2 (cat. no. 122372); human xCT siRNA (cat. no. 108518); and negative control siRNA (cat. no. AM4611) were purchased from Life Technologies (Thermo Fisher Scientific, Inc.). Tca8113 cells were seeded in 12 -well plates at a density of $1.5 \times 10^{5}$ cells/well.. The following day, the cells were transfected with siRNA using Lipofectamine RNAiMax (Life Technologies; Thermo Fisher Scientific, Inc.). Upon $24 \mathrm{~h}$ incubation, the transfected cells were treated with CDDP for the indicated concentrations and times.

Cell viability assay. Tca8113 cells were seeded in 96-well plates $\left(1.5 \times 10^{4}\right.$ cells/well $)$ prior to be subjected to transfection with $\mathrm{xCT}$, Nrf2 or ATF4 siRNA, or to treatment with $\mathrm{SASP} / \beta$-ME for $24 \mathrm{~h}$, followed by incubation with $5,10,20$, 30 or $40 \mu \mathrm{g} / \mathrm{ml}$ CDDP for an additional $48 \mathrm{~h}$. Cell viability was measured using Cell Counting Kit-8 (CCK-8) (Sigma-Aldrich). Briefly, the culture medium was replaced following CDDP treatment, and $10 \mu \mathrm{l} \mathrm{CCK}-8$ was added. Following incubation for $45 \mathrm{~min}$ at $37^{\circ} \mathrm{C}$, the absorbance was measured at a wavelength of $450 \mathrm{~nm}$ using a microplate reader (Tecan Group Ltd., Männedorf, Switzerland).

Statistical analysis. Data are presented as the mean \pm standard error of the mean. Statistical analysis was conducted using one-way analysis of variance. All statistical analyses were performed using SPSS 18.0 statistical software (SPSS, Inc., Chicago, IL, USA). $\mathrm{P}<0.05$ was considered to indicate a statistically significant difference.

\section{Results}

CDDP induces $x$ CT expression in Tca8113 cells. To elucidate the function of $\mathrm{xCT}$ in CDDP resistance, CDDP-inducible messenger RNA (mRNA) and protein $\mathrm{xCT}$ expression levels were investigated. As indicated in Fig. 1A-C, CDDP significantly increased $\mathrm{xCT}$ mRNA and protein expression levels in a time- and concentration-dependent manner, and $20 \mu \mathrm{g} / \mathrm{ml} \mathrm{CDDP}$ induced maximal xCT expression following 6-h treatment. Therefore, this concentration of CDDP and treatment time was selected for subsequent experiments. CDDP induced $\mathrm{xCT}$ upregulation and a marginal increase in the expression of the heavy chain of system $\mathrm{x}_{\mathrm{c}}{ }^{-}$(4F2hc/CD98) $4 \mathrm{~F} 2 \mathrm{hc}$, which may be due to a positive feedback as a consequence of $\mathrm{xCT}$ induction (Fig. 1A-C). Ishimoto et al (33) indicated that $\mathrm{CD} 44 \mathrm{v}$ interacts with and stabilizes $\mathrm{xCT}$, thus regulating the redox status and promoting tumor growth, however no significant differences in CD44v expression were observed in the present study following CDDP treatment in Tca8113 cells (Fig. 1C and D). These results demonstrated that CDDP treatment significantly increased $\mathrm{xCT}$ expression in Tca8113 cells.

CDDP enhances $x C T$ expression in an Nrf2 and ATF4-dependent manner. Firstly, to clarify whether CDDP-induced xCT expression was dependent on Nrf2 and ATF4, the knockdown efficiency of siRNA transfection was confirmed. As shown in Fig. 2A and B, Nrf2 and ATF4 expression was effectively inhibited by individual siRNAs in the DMSO- and CDDP-treated Tca8113 cells. Two types of siRNA, which targeted Nrf2 or ATF4, were used to prevent potential off-target effects (silencing of genes other than Nrf2 and ATF4) arising from non-specific binding of the siRNAs to unrelated genes. The results demonstrated that CDDP-induced $\mathrm{xCT}$ expression was significantly decreased in Nrf2 and ATF4 knockdown cells (Fig. 2C and D). These results indicate that $\mathrm{CDDP}$-induced $\mathrm{xCT}$ expression occurs in a Nrf2 and ATF4-dependent manner.

CDDP induces $x C T$ activation via ARE and AARE elements on the promoter of the human $x C T$ gene. The function of the cis elements on the promoter of human $x C T$ in CDDP-induced $\mathrm{xCT}$ expression was investigated using a series of $\mathrm{xCT}$ gene promoter luciferase reporter plasmids. As represented in Fig. 3, CDDP induced reporter activity in the wild-type construct. Notably, constitutive and CDDP-inducible reporter 
A

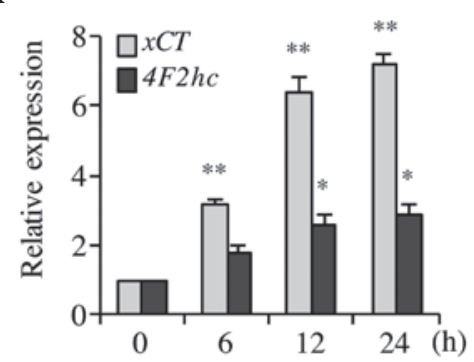

C

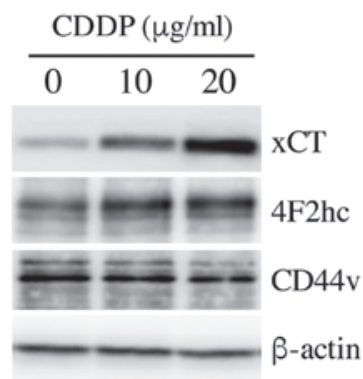

B

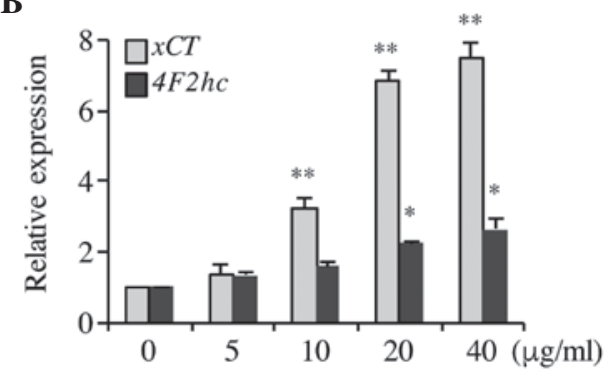

D

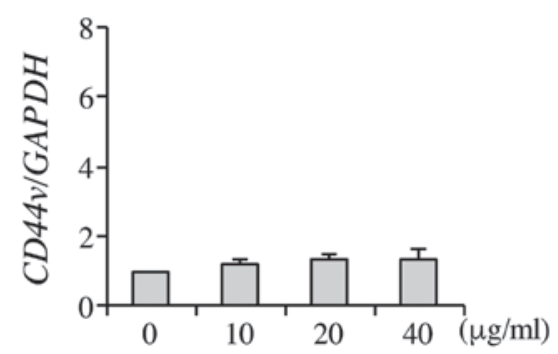

Figure 1. CDDP induced $\mathrm{xCT}$ expression at the mRNA and protein levels in Tca8113 cells. (A) Tca8113 cells were treated with $20 \mu \mathrm{g} / \mathrm{ml} \mathrm{CDDP}$ for $24 \mathrm{~h}$. Tca8113 cells were subjected to treatment with increasing concentrations of CDDP as indicated for $12 \mathrm{~h} . x C T$ and $4 F 2 h c$ (B) mRNA and (C) protein expression levels were determined by RT-qPCR and immunoblotting, respectively. (D) CD44v mRNA expression was determined by RT-qPCR. Data are presented as the mean \pm standard error of the mean of $\geq 3$ independent experiments. ${ }^{*} \mathrm{P}<0.05,{ }^{* *} \mathrm{P}<0.01$ vs. control. CDDP, cisplatin; RT-qPCR, reverse transcription-quantitative polymerase chain reaction; CD44v, cluster of differentiation 44 variant; GAPDH, glyceraldehyde-3-phosphate dehydrogenase; mRNA, messenger RNA.

A

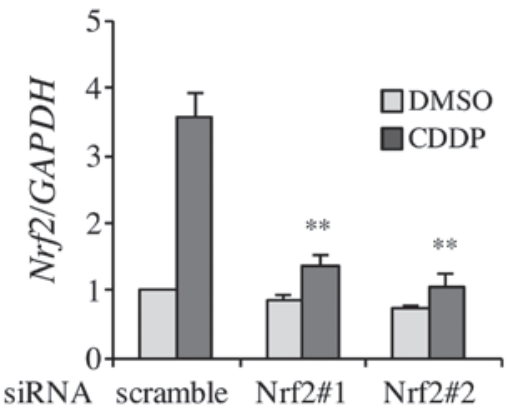

C

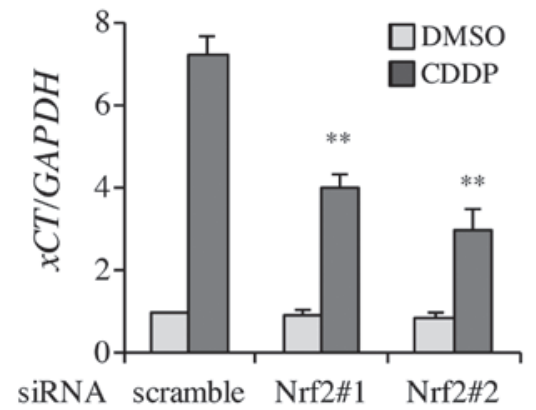

B

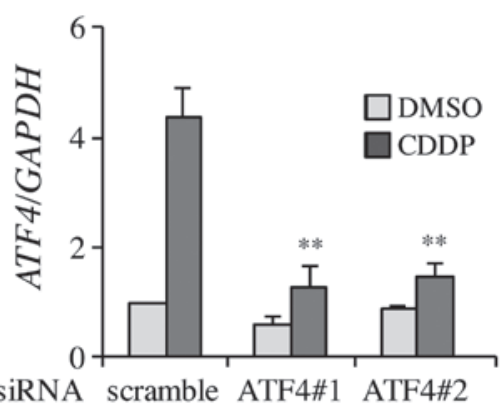

D

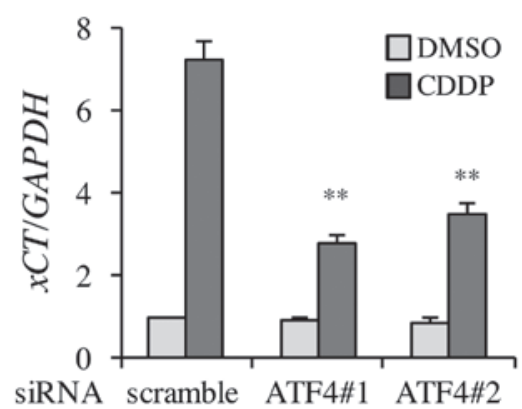

Figure 2. Nrf2 and ATF4 knockdown attenuated CDDP-induced xCT expression. (A and B) Tca8113 cells were transfected with siRNAs targeting (A) Nrf2 or (B) ATF4. Upon $24 \mathrm{~h}$, cells were treated with $20 \mu \mathrm{g} / \mathrm{ml}$ CDDP for $12 \mathrm{~h}$. The expression of xCT, Nrf2 and ATF4 messenger RNA was then measured using reverse transcription-quantitative polymerase chain reaction. (C and D) Tca8113 cells were transfected with siRNAs targeting (C) Nrf2 or (D) ATF4, and then treated with CDDP as described above. The expression levels of xCT was subsequently measured. Data are presented as the mean \pm standard error of the mean of $\geq 3$ independent experiments. ${ }^{* *} \mathrm{P}<0.01$ vs. control. CDDP, cisplatin; Nrf2, nuclear factor erythroid 2-related-factor 2; ATF4, activating transcription factor 4; DMSO, dimethyl sulfoxide; siRNA, small interfering RNA; GAPDH, glyceraldehyde-3-phosphate dehydrogenase.

activities were decreased in ARE, AARE-F and AARE-R mutant reporter genes, and this effect was decreased further in the AARE-F\&R mutant reporter construct. CDDP-inducible reporter activity was almost eliminated in the triple mutant reporter gene. These results indicate that ARE and AARE are required for $\mathrm{CDDP}$-triggered $\mathrm{xCT}$ induction. 


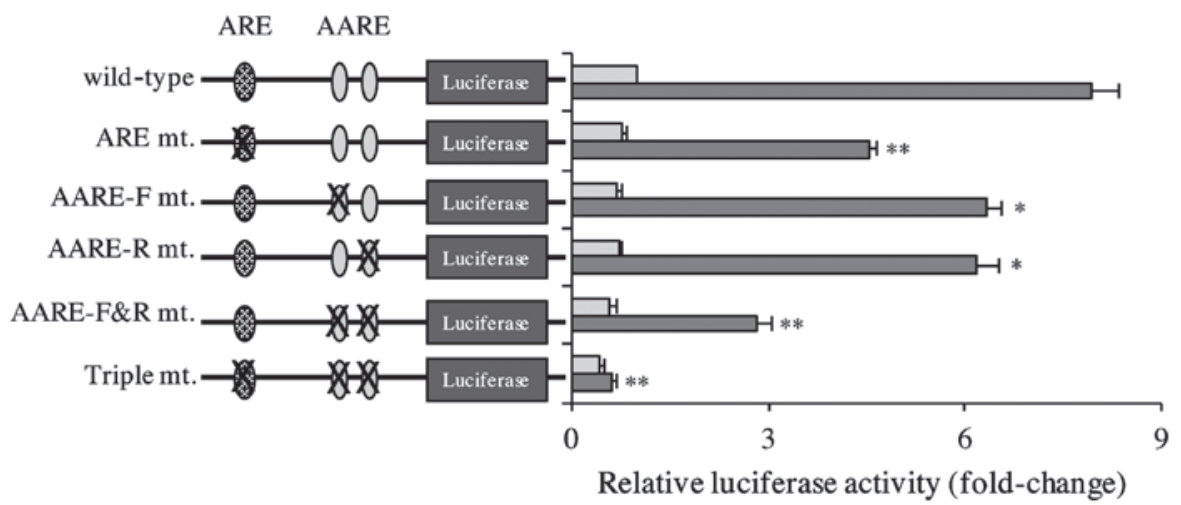

Figure 3. CDDP-inducible $\mathrm{xCT}$ activation was dependent on the ARE and AARE on the promoter of the human $x C T$ gene. Tca8113 cells were transfected with wild-type or mutant XCT promoter luciferase reporter plasmids, and $24 \mathrm{~h}$ later, the culture medium was replaced with $20 \mu \mathrm{g} / \mathrm{ml} \mathrm{CDDP}$. Following $24 \mathrm{~h}$, the reporter activity was determined. Data are presented as the mean \pm standard error of the mean. ${ }^{*} \mathrm{P}<0.05,{ }^{* *} \mathrm{P}<0.01$ vs. control. ARE, antioxidant response element; AARE, amino acid response element; CDDP, cisplatin; F, forward; R, reverse; mt., mutant.

A

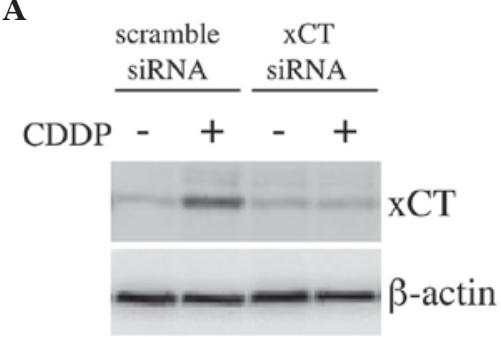

C

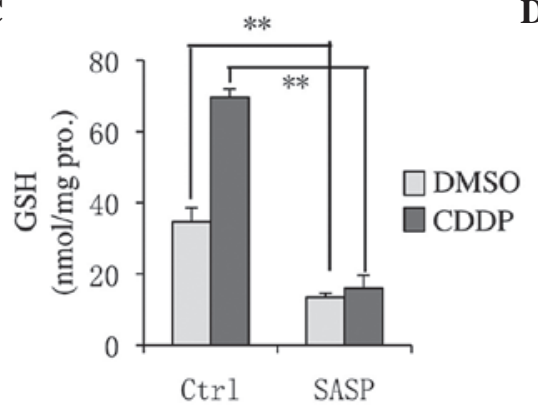

$\mathbf{E}$

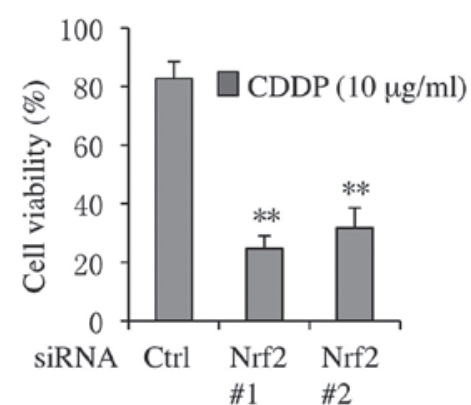

F
B

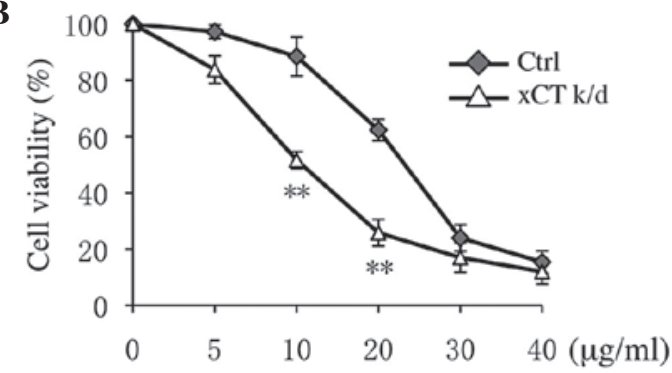

D
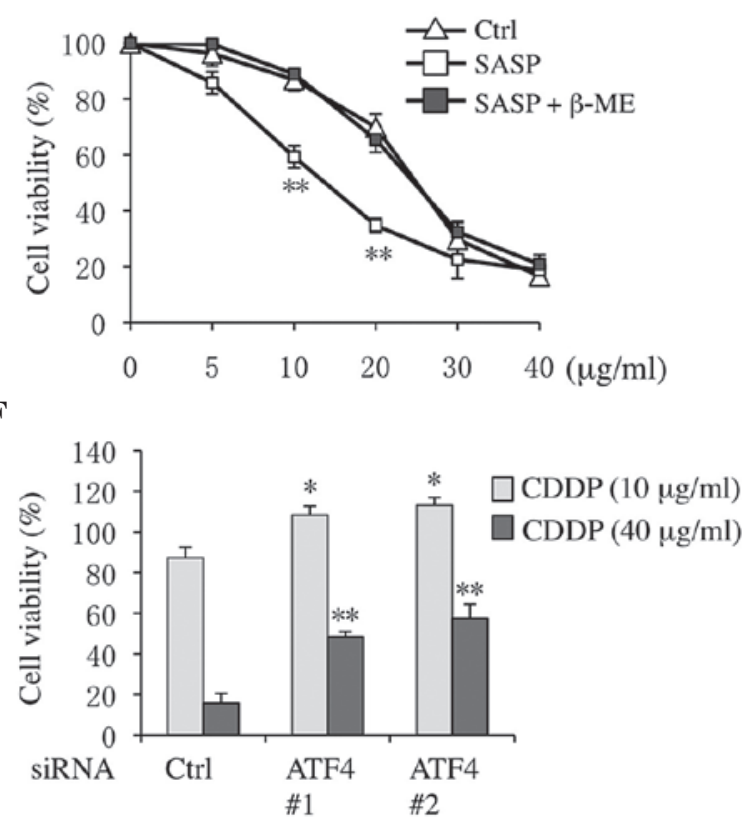

Figure 4. xCT inhibition sensitized Tca8113 cells to CDDP. (A) Tca8113 cells were treated with $20 \mu \mathrm{g} / \mathrm{ml}$ CDDP for $12 \mathrm{~h}$ following transfection with $\mathrm{xCT}$ or scramble siRNA. (B) Tca8113 cells were transfected with xCT or scramble siRNA for $24 \mathrm{~h}$, and cells were next incubated with $0,5,10,20,30 \mathrm{or} 40 \mu \mathrm{g} / \mathrm{ml}$ CDDP for an additional $48 \mathrm{~h}$. Cell viability was measured with the Cell Counting Kit- 8 assay. (C) Tca8113 cells were pre-treated with $0.3 \mathrm{mM}$ SASP for $30 \mathrm{~min}$, and then cultured with $20 \mu \mathrm{g} / \mathrm{ml} \mathrm{CDDP}$ for $24 \mathrm{~h}$. Subsequently, intercellular glutathione levels were estimated. (D) Tca8113 cells were treated with SASP and 0-40 $\mu \mathrm{g} / \mathrm{ml} \mathrm{CDDP} \mathrm{for} 48 \mathrm{~h}$, and cell viability was analyzed. Cells were transfected with (E) Nrf2 or (F) ATF4 siRNAs for $24 \mathrm{~h}$, and then subjected to 10 or $40 \mu \mathrm{g} / \mathrm{ml}$ CDDP treatment for an additional $48 \mathrm{~h}$. Data are presented as the mean \pm standard error of the mean. " $\mathrm{P}<0.05$, ${ }^{* *} \mathrm{P}<0.01 \mathrm{vs}$. control. CDDP, cisplatin; siRNA, small interfering RNA; SASP, sulfasalazine; Nrf2, nuclear factor erythroid 2-related-factor 2; ATF4, activating transcription factor 4; $\beta$-ME, $\beta$-mercaptoethanol; Ctrl, control; GSH, glutathione; DMSO, dimethyl sulfoxide; k/d, knockdown.

xCT knockdown or inhibition increases Tca8113 cell sensitivity to CDDP. Firstly, the siRNA knockdown efficiency for targeting human $\mathrm{xCT}$ was confirmed. $\mathrm{xCT}$ expression levels were effectively decreased in both DMSO- and CDDP-treated cells (Fig. 4A). Notably, CDDP cytotoxicity was markedly increased in XCT knockdown cells compared with scramble 


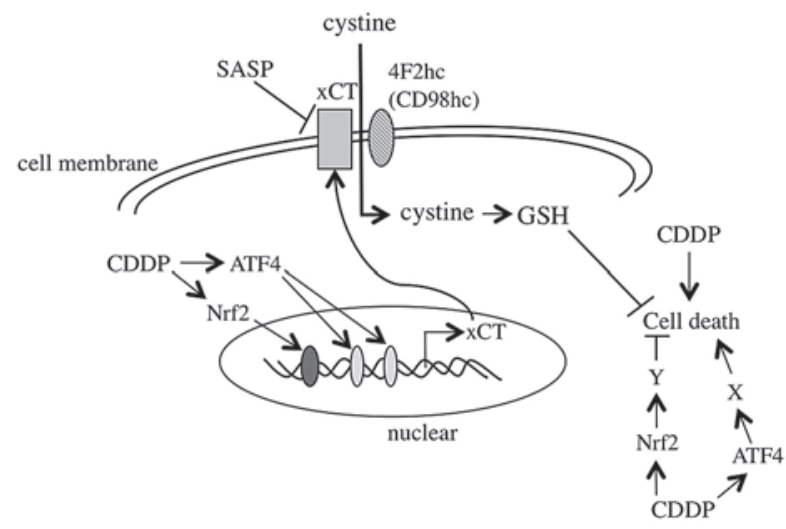

Figure 5. Hypothetical model of CDDP-induced xCT activation pathway and the mechanism of tongue squamous cell carcinoma CDDP resistance. CDDP, cisplatin; Nrf2, nuclear factor erythroid 2-related factor 2; ATF4, activating transcription factor 4; GSH, glutathione; SASP, sulfasalazine; CD98, cluster of differentiation 98 .

siRNA-transfected cells (Fig. 4B). Similarly, following co-treatment with DMSO or CDDP and the xCT pharmacological inhibitor SASP, the levels of GSH were downregulated in both DMSO- and CDDP-treated cells (Fig. 4C). Furthermore, co-treatment with SASP and CDDP markedly sensitized Tca8113 cells to CDDP (Fig. 4D). The inhibitory effect of SASP was suppressed following the addition of $\beta$-ME, which could bypass $\mathrm{XCT}$ to allow cysteine uptake via natural amino acid transporters (19). Previously, it has been demonstrated that Nrf2 and ATF4 regulate $\mathrm{xCT}$ following CDDP treatment $(15,18)$. Thus, the effect of Nrf2 and ATF4 knockdown on the sensitivity of Tca8113 cells to CDDP was also assessed in the present study. As shown in Fig. 4E, CDDP inducible cytotoxicity was markedly increased in Nrf2 knockdown cells compared with the control. By contrast, following ATF4 knockdown, cell viability increased in both high and low CDDP concentration-treatment groups compared with the control (Fig. 4F).

\section{Discussion}

Chemotherapy is a widely used cancer treatment, in addition to surgery and radiotherapy (3). CDDP is the standard chemotherapeutic drug administered to patients with tongue carcinoma (3). However, drug resistance is common and leads to the failure of CDDP therapy (10). Thus, the mechanisms underlying tongue carcinoma cell resistance to CDDP require investigation. An increasing number of studies have indicated that CDDP resistance is associated with various factors, including various microRNAs, which modulate CDDP chemosensitivity by targeting certain genes $(34,35)$; cell protective autophagy, which diminishes CDDP-induced apoptotic cell death (36); upregulated GSH levels; and downregulated ROS levels $(15,37)$. In the present study, the mechanism of CDDP-triggered $\mathrm{xCT}$ induction and the function of $\mathrm{xCT}$ in tongue carcinoma cell CDDP resistance was investigated, and the conclusion is summarized in Fig. 5.

$\mathrm{xCT}$ is essential for GSH synthesis and maintenance, which is a critical regulator of the cellular redox state (38). Thus, $\mathrm{xCT}$ has been considered as a potential target for cancer treatment, including tongue carcinoma (24). Previous studies have demonstrated that SASP enhances CDDP-inducible cytotoxicity by preventing electrophiles from conjugating with GSH, indicating that combined treatment with CDDP and SASP may be beneficial (31). SASP is a well-established pharmacological inhibitor of $\mathrm{xCT}$, however, the effect of SASP treatment on $\mathrm{xCT}$ and GSH levels has not yet been investigated $(31,39)$. In the present study, xCT suppression increased CDDP cytotoxicity. In addition, CDDP treatment markedly increased GSH levels in Tca8113 tongue cancer cells, which was attenuated by SASP-induced xCT suppression (Fig. 4C). Furthermore, the sensitivity of Tca8113 cells to CDDP treatment increased under conditions of low GSH levels as a result of $\mathrm{xCT}$ inhibition caused by SASP or siRNA (Fig. 4B and D). However, the present study did not provide immediate evidence that decreased GSH levels trigger ROS accumulation and subsequent activation of the cell death signaling pathway in CDDP and SASP co-treated Tca8113 cells, although previous studies have suggested such association in various experimental conditions (40-42).

It has been reported that $\mathrm{xCT}$ is modulated in an Nrf2 and ATF4-dependent manner following proteasome inhibitor treatment (19). In the present study, Nrf2 and ATF4 knockdown by siRNAs attenuated CDDP-triggered $\mathrm{xCT}$ induction (Fig. 2). Furthermore, the binding sites ARE and AARE of Nrf2 and ATF4 are critical for CDDP-inducible XCT activation (Fig. 3). Additionally, no significant differences in the mRNA or protein expression levels of CD44v were identified following CDDP treatment in Tca8113 cells, although previous studies have indicated that CD44v may promote tumor growth by stabilizing $\mathrm{xCT}$ (33) and increasing CDDP resistance in head and neck squamous cells (43). It is hypothesized that $\mathrm{CD} 44 \mathrm{v}$ may stabilize $\mathrm{xCT}$; however, the association between CDDP treatment and $\mathrm{CD} 44 \mathrm{v}$ induction remains unclear. The present authors hypothesize that CDDP-inducible $\mathrm{xCT}$ activation predominantly occurs via binding of Nrf2 and ATF4 to the ARE and AARE elements on the promoter of the $\mathrm{xCT}$ gene, rather than as a result of increased stabilization of the $\mathrm{xCT}-\mathrm{CD} 44 \mathrm{v}$ complex. Although the present study demonstrated that $\mathrm{xCT}$ is modulated by Nrf2 and ATF4 and contributes to the resistance of CDDP treatment in Tca8113 cells, the effect of Nrf2 and ATF4 suppression by siRNA was not consistent with the effects of xCT knockdown (Fig. 4E and F). Previously, Tanabe et al (26) reported that CDDP-inducible upregulation of ATF4 increased CDDP resistance in human KB epidermoid and prostate cancer cell lines. However, in the present study, Tca8113 cells treated with 10 or $40 \mu \mathrm{g} / \mathrm{ml}$ CDDP exhibited lower sensitivity to CDDP compared with the control following ATF4 knockdown (Fig. 4F). It is possible to hypothesize that an alternative gene, in addition to $\mathrm{xCT}$, is downregulated in the downstream ATF4 signaling cascade, such as the C/EBP homologous protein (CHOP) (44). CHOP induction is the key factor in ER stress-induced apoptosis $(45,46)$, and is also a repressor of Wnt/T-cell factor signaling (47), which is associated with angiogenesis, migration and survival of cancer cells (48-50). However, in the present study, the suppression of Nrf2 by siRNA exhibited the opposite effect to ATF4 suppression. It was demonstrated that Nrf2 knockdown sensitized Tca8113 cells to CDDP and exceeded the effects of XCT knockdown 
(Fig. 4E). Nrf2 exhibits a critical function in the induction of phase II detoxifying enzymes, including glutamate-L-cysteine ligase catalytic subunit, nicotinamide adenine dinucleotide (P) H: quinone oxidoreductase and heme oxygenase-1, via the Kelch-like ECH-associated protein 1-Nrf2-ARE pathway, which has been reported to induce CDDP resistance in tumor cells $(51,52)$. Although Nrf2 and its target gene contribute to CDDP resistance, the present authors postulate that it is not advisable to increase CDDP cytotoxicity via Nrf2 suppression. In various animal models (53-55), Nrf2-ARE activation has been demonstrated to prevent CDDP-induced nephrotoxicity, which is the most severe adverse effect that limits high-dose CDDP therapy (56).

In conclusion, the present study demonstrated that Nrf2/ATF4-dependent $\mathrm{xCT}$ induction is involved in CDDP resistance of Tca8113 tongue carcinoma cells. These results suggest that it may be beneficial to combine CDDP and pharmacological $\mathrm{xCT}$ inhibitors for the treatment of tongue carcinoma.

\section{References}

1. Siegel R, Naishadham D and Jemal A: Cancer statistics, 2012. CA Cancer J Clin 62: 10-29, 2012.

2. Jemal A, Bray F, Center MM, Ferlay J, Ward E and Forman D: Global cancer statistics. CA Cancer J Clin 61: 69-90, 2011.

3. O'Neill VJ and Twelves CJ: Oral cancer treatment: Developments in chemotherapy and beyond. Br J Cancer 87: 933-937, 2002.

4. Gibson MK and Forastiere AA: Reassessment of the role of induction chemotherapy for head and neck cancer. Lancet Oncol 7: 565-574, 2006

5. Tsukada H, Yokoyama A, Goto K, Shinkai T, Harada M, Ando M, Shibata T, Ohe Y, Tamura T and Saijo N; Lung Cancer Study Group of the Japan Clinical Oncology Group (JCOG) Randomized controlled trial comparing docetaxel-cisplatin combination with weekly docetaxel alone in elderly patients with advanced non-small-cell lung cancer: Japan Clinical Oncology Group (JCOG) 0207. Jpn J Clin Oncol 45: 88-95, 2015.

6. Li W, Wan L, Zhai LY and Wang J: Effects of SC-560 in combination with cisplatin or taxol on angiogenesis in human ovarian cancer xenografts. Int J Mol Sci 15: 19265-19280, 2014.

7. Hu MH, Wang LW, Lu HJ, Chu PY, Tai SK, Lee TL, Chen MH, Yang MH and Chang PM: Cisplatin-based chemotherapy versus cetuximab in concurrent chemoradiotherapy for locally advanced head and neck cancer treatment. BioMed Res Int 2014: 904341, 2014

8. Wang L, Zhang Y, Zhao J, Xiao E, Lu J, Fu S and Wang Z: Combination of bladder cancer-specific oncolytic adenovirus gene therapy with cisplatin on bladder cancer in vitro. Tumour Biol 35: 10879-10890, 2014.

9. Wang B, Zhang S, Yue K and Wang XD: The recurrence and survival of oral squamous cell carcinoma: A report of 275 cases. Chin J Cancer 32: 614-618, 2013.

10. Galluzzi L, Senovilla L, Vitale I, Michels J, Martins I, Kepp O, Castedo M and Kroemer G: Molecular mechanisms of cisplatin resistance. Oncogene 31: 1869-1883, 2012.

11. Wang X, Martindale JL and Holbrook NJ: Requirement for ERK activation in cisplatin-induced apoptosis. J Biol Chem 275: 39435-39443, 2000

12. Mandic A, Hansson J, Linder S and Shoshan MC: Cisplatin induces endoplasmic reticulum stress and nucleus-independent apoptotic signaling. J Biol Chem 278: 9100-9106, 2003.

13. Zhou XJ, Chen WT, Li Q and He RG: Establishment and biological characteristics of cisplatin resistant cell line from human tongue squamous cell carcinoma Tca8113. Shanghai Kou Qiang Yi Xue 10: 31-34, 2001 (In Chinese).

14. Lu SC: Glutathione synthesis. Biochim Biophys Acta 1830 3143-3153, 2013

15. Okuno S, Sato H, Kuriyama-Matsumura K, Tamba M, Wang H, Sohda S, Hamada H, Yoshikawa H, Kondo T and Bannai S: Role of cystine transport in intracellular glutathione level and cisplatin resistance in human ovarian cancer cell lines. Br J Cancer 88: 951-956, 2003
16. Wangpaichitr M, Sullivan EJ, Theodoropoulos G, Wu C, You M, Feun LG, Lampidis TJ, Kuo MT and Savaraj N: The relationship of thioredoxin-1 and cisplatin resistance: Its impact on ROS and oxidative metabolism in lung cancer cells. Mol Cancer Ther 11: 604-615, 2012.

17. Wangpaichitr M, Wu C, You M, Maher JC, Dinh V, Feun LG and Savaraj N: N',N'-Dimethyl-N',N'-bis(phenylcarbonothioyl) propanedihydrazide (elesclomol) selectively kills cisplatin resistant lung cancer cells through reactive oxygen species (ROS). Cancers (Basel) 1: 23-38, 2009.

18. Sato H, Tamba M, Ishii $\mathrm{T}$ and Bannai $\mathrm{S}$ : Cloning and expression of a plasma membrane cystine/glutamate exchange transporter composed of two distinct proteins. J Biol Chem 274: 11455-11458, 1999.

19. Ye P, Mimura J, Okada T, Sato H, Liu T, Maruyama A, Ohyama C and Itoh K: Nrf2- and ATF4-dependent upregulation of xCT modulates the sensitivity of T24 bladder carcinoma cells to proteasome inhibition. Mol Cell Biol 34: 3421-3434, 2014.

20. Wada T, Ishimoto T, Seishima R, Tsuchihashi K, Yoshikawa M, Oshima H, Oshima M, Masuko T, Wright NA, Furuhashi S, et al: Functional role of CD44v-xCT system in the development of spasmolytic polypeptide-expressing metaplasia. Cancer Sci 104: 1323-1329, 2013.

21. Takeuchi S, Wada K, Toyooka T, Shinomiya N, Shimazaki H, Nakanishi K, Nagatani K, Otani N, Osada H, Uozumi Y, et al: Increased $\mathrm{xCT}$ expression correlates with tumor invasion and outcome in patients with glioblastomas. Neurosurgery 72: 33-41, 2013.

22. Huang Y, Dai Z, Barbacioru C and Sadée W: Cystine-glutamate transporter SLC7A11 in cancer chemosensitivity and chemoresistance. Cancer Res 65: 7446-7454, 2005.

23. Savaskan NE, Heckel A, Hahnen E, Engelhorn T, Doerfler A, Ganslandt O, Nimsky C, Buchfelder M and Eyüpoglu IY: Small interfering RNA-mediated xCT silencing in gliomas inhibits neurodegeneration and alleviates brain edema. Nat Med 14: 629-632, 2008

24. Toyoda M,Kaira K, Ohshima Y,Ishioka NS, Shino M, Sakakura K, Takayasu Y, Takahashi K, Tominaga H, Oriuchi N, et al: Prognostic significance of amino-acid transporter expression (LAT1, ASCT2, and $\mathrm{xCT}$ ) in surgically resected tongue cancer. Br J Cancer 110: 2506-2513, 2014.

25. El-Sawalhi MM and Ahmed LA: Exploring the protective role of apocynin, a specific NADPH oxidase inhibitor, in cisplatin-induced cardiotoxicity in rats. Chem Biol Interact 207: 58-66, 2014.

26. Tanabe M, Izumi H, Ise T, Higuchi S, Yamori T, Yasumoto K and Kohno K: Activating transcription factor 4 increases the cisplatin resistance of human cancer cell lines. Cancer Res 63: 8592-8595, 2003.

27. Lo M, Wang YZ and Gout PW: The $\mathrm{xc}^{-}$cystine/glutamate antiporter: A potential target for therapy of cancer and other diseases. J Cell Physiol 215: 593-602, 2008.

28. Guo W, Zhao Y, Zhang Z, Tan N, Zhao F, Ge C, Liang L, Jia D, Chen T, Yao M, et al: Disruption of xCT inhibits cell growth via the ROS/autophagy pathway in hepatocellular carcinoma. Cancer Lett 312: 55-61, 2011.

29. Huang Z, Guo KJ, Guo RX and He SG: Effects of 5-fluouracil combined with sulfasalazine on human pancreatic carcinoma cell line BxPC-3 proliferation and apoptosis in vitro. Hepatobiliary Pancreat Dis Int 6: 312-320, 2007.

30. Lay JD, Hong CC, Huang JS, Yang YY, Pao CY, Liu CH, Lai YP, Lai GM, Cheng AL, Su IJ and Chuang SE: Sulfasalazine suppresses drug resistance and invasiveness of lung adenocarcinoma cells expressing AXL. Cancer Res 67: 3878-3887, 2007.

31. Awasthi S, Sharma R, Singhal SS, Herzog NK, Chaubey M and Awasthi YC: Modulation of cisplatin cytotoxicity by sulphasalazine. Br J Cancer 70: 190-194, 1994.

32. Newton GL, Dorian R and Fahey RC: Analysis of biological thiols: Derivatization with monobromobimane and separation by reverse-phase high-performance liquid chromatography. Anal Biochem 114: 383-387, 1981.

33. Ishimoto $T$, Nagano $O$, Yae $T$, Tamada M, Motohara $T$, Oshima H, Oshima M, Ikeda T, Asaba R, Yagi H, et al: CD44 variant regulates redox status in cancer cells by stabilizing the $\mathrm{xCT}$ subunit of system $\mathrm{xc}^{(-)}$and thereby promotes tumor growth. Cancer Cell 19: 387-400, 2011.

34. Ren W, Wang X, Gao L, Li S, Yan X, Zhang J, Huang C, Zhang Y and Zhi K: MiR-21 modulates chemosensitivity of tongue squamous cell carcinoma cells to cisplatin by targeting PDCD4. Mol Cell Biochem 390: 253-262, 2014. 
35. Qin J, Luo M, Qian H and Chen W: Upregulated miR-182 increases drug resistance in cisplatin-treated HCC cell by regulating TP53INP1. Gene 538: 342-347, 2014.

36. Zhang N, Dai L, Qi Y, Di W and Xia P: Combination of FTY720 with cisplatin exhibits antagonistic effects in ovarian cancer cells: Role of autophagy. Int J Oncol 42: 2053-2059, 2013.

37. Li Y, Li X, Wong YS, Chen T, Zhang H, Liu C and Zheng W: The reversal of cisplatin-induced nephrotoxicity by selenium nanoparticles functionalized with 11-mercapto-1-undecanol by inhibition of ROS-mediated apoptosis. Biomaterials 32: 9068-9076, 2011.

38. Barros MP, Marin DP, Bolin AP, de Cássia Santos Macedo R, Campoio TR, Fineto C Jr, Guerra BA, Polotow TG, Vardaris C, Mattei R and Otton R: Combined astaxanthin and fish oil supplementation improves glutathione-based redox balance in rat plasma and neutrophils. Chem Biol Interact 197: 58-67, 2012.

39. Gout PW, Buckley AR, Simms CR and Bruchovsky N: Sulfasalazine, a potent suppressor of lymphoma growth by inhibition of the $\mathrm{x}(\mathrm{c})^{-}$cystine transporter: A new action for an old drug. Leukemia 15: 1633-1640, 2001.

40. You BR and Park WH: Arsenic trioxide induces human pulmonary fibroblast cell death via increasing ROS levels and GSH depletion. Oncol Rep 28: 749-757, 2012.

41. Park WH and Kim SH: MG132, a proteasome inhibitor, induces human pulmonary fibroblast cell death via increasing ROS levels and GSH depletion. Oncol Rep 27: 1284-1291, 2012.

42. You BR and Park WH: Suberoyl bishydroxamic acid-induced apoptosis in HeLa cells via ROS-independent, GSH-dependent manner. Mol Biol Rep 40: 3807-3816, 2013.

43. Torre C, Wang SJ, Xia W and Bourguignon LY: Reduction of hyaluronan-CD44-mediated growth, migration, and cisplatin resistance in head and neck cancer due to inhibition of Rho kinase and PI-3 kinase signaling. Arch Otolaryngol Head Neck Surg 136: 493-501, 2010.

44. Cao J, Dai DL, Yao L, Yu HH, Ning B, Zhang Q, Chen J, Cheng WH, Shen W and Yang ZX: Saturated fatty acid induction of endoplasmic reticulum stress and apoptosis in human liver cells via the PERK/ATF4/CHOP signaling pathway. Mol Cell Biochem 364: 115-129, 2012.

45. Zinszner H, Kuroda M, Wang X, Batchvarova N, Lightfoot RT, Remotti H, Stevens JL and Ron D: CHOP is implicated in programmed cell death in response to impaired function of the endoplasmic reticulum. Genes Dev 12: 982-995, 1998.
46. Oyadomari S and Mori M: Roles of CHOP/GADD153 in endoplasmic reticulum stress. Cell Death Differ 11: 381-389, 2004.

47. Horndasch M, Lienkamp S, Springer E, Schmitt A, Pavenstädt H, Walz $\mathrm{G}$ and Gloy J: The C/EBP homologous protein CHOP (GADD153) is an inhibitor of Wnt/TCF signals. Oncogene 25: 3397-3407, 2006.

48. Tetsu $\mathrm{O}$ and McCormick F: Beta-catenin regulates expression of cyclin D1 in colon carcinoma cells. Nature 398: 422-426, 1999.

49. Zhang X, Gaspard JP and Chung DC: Regulation of vascular endothelial growth factor by the Wnt and K-ras pathways in colonic neoplasia. Cancer Res 61: 6050-6054, 2001.

50. Fujita M, Furukawa Y, Tsunoda T, Tanaka T, Ogawa M and Nakamura Y: Up-regulation of the ectodermal-neural cortex 1 (ENC1) gene, a downstream target of the beta-catenin/T-cell factor complex, in colorectal carcinomas. Cancer Res 61: 7722-7726, 2001.

51. Bao LJ, Jaramillo MC, Zhang ZB, Zheng YX, Yao M, Zhang DD and Yi XF: Nrf2 induces cisplatin resistance through activation of autophagy in ovarian carcinoma. Int J Clin Exp Pathol 7: $1502-1513,2014$

52. Hayden A, Douglas J, Sommerlad M, Andrews L, Gould K, Hussain S, Thomas GJ, Packham G and Crabb SJ: The Nrf2 transcription factor contributes to resistance to cisplatin in bladder cancer. Urol Oncol 32: 806-814, 2014.

53. Li M, Jin J, Li J, Guan CW, Wang WW, Qiu YW and Huang ZY: Schisandrin B protects against nephrotoxicity induced by cisplatin in HK-2 cells via Nrf2-ARE activation. Yao Xue Xue Bao 47: 1434-1439, 2012 (In Chinese).

54. Sahin K, Tuzcu M, Gencoglu H, Dogukan A, Timurkan M, Sahin N, Aslan A and Kucuk O: Epigallocatechin-3-gallate activates Nrf2/HO-1 signaling pathway in cisplatin-induced nephrotoxicity in rats. Life Sci 87: 240-245, 2010.

55. Aleksunes LM, Goedken MJ, Rockwell CE, Thomale J, Manautou JE and Klaassen CD: Transcriptional regulation of renal cytoprotective genes by Nrf2 and its potential use as a therapeutic target to mitigate cisplatin-induced nephrotoxicity. J Pharmacol Exp Ther 335: 2-12, 2010.

56. Luke DR, Vadiei K and Lopez-Berestein G: Role of vascular congestion in cisplatin-induced acute renal failure in the rat. Nephrol Dial Transplant 7: 1-7, 1992. 